Available online at GSC Online Press Directory

GSC Biological and Pharmaceutical Sciences

e-ISSN: 2581-3250, CODEN (USA): GBPSC2

Journal homepage: https://www.gsconlinepress.com/journals/gscbps

(CASE STUdY)

\title{
Assessment of efficacy of ticagrelor versus clopidogrel in the treatment of myocardial infarction by 2D echocardiography
}

\author{
Marina Gladys D' Souza ${ }^{1,}{ }^{*}$, Aishwarya Goud Muthyala ${ }^{1}$, Tejaswini Myaka ${ }^{1}$, Sanjib Kumar Sahu ${ }^{2}$ and Swathi \\ Boddupalli 3 \\ ${ }^{1}$ Bharat Institute of technology, Department of Pharmacognosy, Mangalpally, Ibrahimpatnam, Hyderabad, India - \\ 501510. \\ 2 Pharm D-Vyear, Department of Pharm D, Bharat School of pharmacy, Mangalpally, Ibrahimpatnam, Hyderabad, India- \\ 501510 \\ ${ }^{3}$ Assistant professor, Department of Pharm D, Bharat School of pharmacy, Mangalpally, Ibrahimpatnam, Hyderabad, \\ India- 501510. \\ ${ }^{4}$ Interventional cardiologist, Department of Cardiology, Durgabhai Deshmukh Hospital and Research Centre, Vidya \\ Nagar, Hyderabad, India.
}

Publication history: Received on 07 August 2020; revised on 31 August 2020; accepted on 03 September 2020

Article DOI: https://doi.org/10.30574/gscbps.2020.12.3.0256

\begin{abstract}
Myocardial infarction (MI) is the irreversible death of heart muscle due to prolonged lack of supply of oxygen resulting in decreased coronary blood flow and if untreated may lead to myocardial damage. Myocardial infarction may be due to several causes viz., coronary occlusion, ventricular hypertrophy, coronary artery emboli or coronary trauma. Drugs used for the treatment of MI includes vasodilators to dilate the arteries and veins, antiarrythmics, thrombolytics to dissolve clots, anti-thrombolytics to prevent thrombus formation, anti-platelet drugs, analgesics to reduce pain, cardiac depressants to control heart rate and contractions. Present study was undertaken to evaluate the efficacy of Ticagrelor $\mathrm{v} / \mathrm{s}$ Clopidogrel the antiplatelet drugs indicated in patients to reduce the risk of myocardial infarction. Both the drugs act by preventing the formation of platelet clots and helps in the smooth flow of blood. Assessment of results was carried out by $2 \mathrm{~d}$ Echo test to evaluate the efficacy of the treatment. Treatment with ticagrelor showed improved efficacy and safety by improving the ejection fraction, reducing the rate of stroke, reducing vascular abnormalities, showing no bleeding problems. No death is observed due to vascular problems in both the treatment. Ticagrelor revealed considerable recovery rate compared to clopidogrel. Study reports revealed that ticagrelorisefficient thanclopidogrel in the treatment of MI and is a better choice over clopidogrel to treat MI.
\end{abstract}

Keywords: Myocardial infarction; Anti-platelet; Ticagrelor; Clopidogrel; 2D echo

\section{Introduction}

Myocardial infarction commonly referred to as a heart attack is often caused by reduced supply of blood flow or oxygen to the heart, resulting in the necrosis of cardiac muscle. This happens usually as a result of blood clot in the epicardial artery, the artery which supplies blood to the tertiary of heart muscle [1]. The prevalence approaches around three million people worldwide ever year. Etiology refers to ruptured atherosclerotic plaques leading to thrombosis, causing decreased blood flow in the coronary artery which is one of the main causes for myocardial infarction. Thrombus formation is regulated by plasma proteins, blood cells and flow and this is fundamental in the development of coronary occlusion. Studies have shown that, thrombi in patients with ST-segment elevated myocardial infarction (STEMI) contain platelets, fibrin and inflammatory blood cells [2]. In non ST-segment elevated myocardial infarction (NSTEMI),

*Corresponding author: Marina Gladys D' Souza

Bharat Institute of technology, Department of Pharmacognosy, Mangalpally, Ibrahimpatnam, Hyderabad, India - 501510. 
atherosclerosis causing partial obstruction of blood flow occurs, resulting in subendocardial ST-depression or unstable angina where as in the case of segment elevated myocardial infarction (STEMI)transmural ST-elevation is observed. Mortality rate due to myocardial infarction has declined due to the introduction of various classes of drugs like anticoagulants, anti-platelet agents, statins and use of revascularization, [3, 4]. But still there is an ambiguity in the choice of specific drug treatment concerning the efficacy and safety of the patients. Studies with respect safety and efficacy of the drugs used to treat MI is still remains a research subject. The present study reveals the efficacy of anti-platelet drugs ticagrelor $\mathrm{v} / \mathrm{s}$ clopidogrel in the treatment of myocardial infarction.

\section{Material and methods}

\subsection{Study design}

The study was initiated after taking the consent from Institutional ethics committee, DurgabaiDeshmukh hospital, Hyderabad. This is a prospective observational study, conducted for six months in the department of cardiology, DurgabaiDeshmukh Hospital from September 2018-March 2019. Patients consent in written format was taken before starting the studies. Number of patients under study was 60. Patients of either sex presenting ST-segment elevated myocardial infarction (STEMI) or Non ST segment elevated myocardial infarction (NSTEMI) with risk rate of 50\% to $85 \%$ ) were selected for the studies. Patients with other co-morbidities like hypertension and diabetes mellitus were also included. Extensive observations of the patients were carried out during six months of study. Baseline demographic data was collected from the patient case reports. Patients with age groups of 30 to 70 were included in the studies. Patients with the age group of less than 30 years, pregnant and lactating women and patients undergoing/underwent surgery were excluded. The entire study was conducted as per AHA/ASA guidelines [5].

\subsection{Assessment of myocardial infarction}

Assessment of extension and severity of MI was carried out by $2 \mathrm{~d}$ Echocardiography [6]. All the patients under treatment were scheduled to undergo $2 \mathrm{~d}$ Echo before and after treatment. Few patients with high risk were suggested take once in two months.

\subsection{Study population}

Sixty patients with STEMI and NSTEMI were selected for the study. Patients were divided into two groups; group A with 35 patients and group B with 25 patients. Group A received Ticagrelor-90 mg/day and Group B received Clopidogrel $75 \mathrm{mg} /$ day- $300 \mathrm{mg}$ based on the condition of the patient. After initial loading dose of $300 \mathrm{mg} / \mathrm{day}$ the dose was slowly reduced to $75 \mathrm{mg} /$ day. Aspirin (75mg to $100 \mathrm{mg} /$ day) was given in combination.

\subsection{Duration of study}

Observational study carried out for six months.

\section{Results}

The present study was undertaken to evaluate the efficacy and safety of ticagrelor versus clopidogrel in the patients with myocardial infarction. The patients with co morbidities like hypertension and diabetes mellitus were also included in the study. 35 patients received ticagrelor and 25 patients received clopidogrel. Comparative analysis of data was carried out by student $t$ test. $\mathrm{P}=0.05$ was taken as significant difference at $95 \% \mathrm{CI}$. As per the results patients treated with ticagrelor showed better improvement with respect to ejection fraction, regional wall motion, right ventricular function and reduced arterial plaques; analyzed by $2 \mathrm{~d}$ echo. Patients did not report any chest pain in both the groups after regular treatment. Clopidogrel showed less improvement in ejection fraction compared to ticagrelor. There was no complaint of bleeding, shock or death of any patients during the treatment period. Aspirin was also co-administered with the drugs under treatment. Even though the difference in efficacy of ticagrelor over clopidogrel was not significant, there was a notable difference in ejection fraction (Table 1). From the study we concluded that ticagrelorcan be preferred over clopidogrel. 
Table 1 Impact of anti- platelet drugs, ticagrelor and clopidogrel in patients with myocardial infarction after six months regular of treatment $(n=30)$.

\begin{tabular}{|c|c|c|c|c|c|}
\hline & \multirow[t]{2}{*}{ Ticagrelor } & \multirow[t]{2}{*}{ Clopidogrel } & \multicolumn{2}{|c|}{$\begin{array}{l}\text { Risk reduction } \% \text { at the } \\
\text { time of testing }\end{array}$} & \multirow[t]{2}{*}{ P-value } \\
\hline & & & Ticagrelor & Clopidogrel & \\
\hline Death & Nil & Nil & 100 & 100 & -- \\
\hline Non-fatal stroke & Nil & Nil & 100 & 100 & -- \\
\hline Anterior STEMI & mild risk & mild risk & 82 & 80 & $\mathrm{P}<0.05$ \\
\hline Regional wall motion & Normal & Near to normal & 80 & 78 & $\mathrm{P}<0.05$ \\
\hline Inferior wall MI & mild risk & mild risk & 77 & 75 & $P<0.05$ \\
\hline NSTEMI & mild risk & Moderately risk & 82 & 72 & $\mathrm{P}>0.05$ \\
\hline $\begin{array}{l}\text { Left ventricular } \\
\text { dysfunction }\end{array}$ & mild risk & mild risk & 84 & 82 & $P<0.05$ \\
\hline Ejection fraction & $\begin{array}{l}\text { More } \\
\text { Significantly } \\
\text { improved }\end{array}$ & $\begin{array}{l}\text { Moderately } \\
\text { improved }\end{array}$ & 82 & 75 & $P<0.05$ \\
\hline
\end{tabular}

Complete remission: $100 \%$ risk reduction (nil) at the time of testing; Mild risk: $<100 \%$ and $\geq 75 \%$ risk reduction; Moderate risk : $\geq 50 \%$ and $<75 \%$ risk reduction; Less improvement: Below 50\% risk reduction ; $\mathrm{P}<0.05$ : No significant difference in efficacy; P $>0.05$ : Significant difference in efficacy

\section{Discussion}

Myocardial infarction (MI) or acute myocardial infarction (AMI) is one of the leading causes of death in the world. MI is due to the lack of oxygen supply to the heart muscle which causes irreversible damage to the heart muscle, if untreated, leading to serious complications.

One of the main reason being atherosclerotic plaques in the blood vessels leading to thrombosis, platelet aggregation, causing decreased blood flow in the coronary artery [7]. The right choice of drug depends upon the risk assessment and development of complications like ischemia and hemorrhage in individual patients. The drugs which inhibit platelet aggregation; ADP P2Y12 blockers: prasugrel, clopidogrel and ticagrelorare one of the choices recommended by world cardiology associations for acute coronary syndrome treatment [8]. Dual therapy with P2Y12 receptor blocker with aspirin is found to be a main therapeutic option to treat MI. Studies have shown that clopidogrel is associated with delayed onset of action and variability in response. Ticagrelor noncompetitive P2Y12 receptor blocker associated with greater platelet inhibition activity with rapid onset of action. The activity was assessed by $2 \mathrm{~d}$ echo. This method is a noninvasive method providing estimation of impaired supply of blood to the myocardium at-a-glance. $2 \mathrm{~d}$ electrocardiography is applied to assess wall motion abnormalities, patient's vascular status, risk stratification, extent of infarction. Doppler electrocardiography provides the information on the status of hemodynamic functions which gives huge therapeutic implications [9]. In this study we have assessed the efficacy of ticagrelor and clopidogrel, on the patients suffering with myocardial infarction. Ticagrelor was found to be better compared to clopidogrel in efficacy as per the $2 \mathrm{~d}$ echo reports. Even-though significant difference was not observed, some notable improvement with respect to ejection fraction and NSTEMI were observed in patients receiving ticagrelor. This finds to be of major therapeutic advantage with ticagrelor.

This delayed action of clopidogrel may be due the impaired generation of active thiol metabolite. Ticagrelor does not require metabolic activation and therefore demonstrates rapidonset effect which may account for the better efficacy of ticagrelor [10].

\section{Conclusion}

Myocardial infarction /Acute myocardial infarction and its related pathologies still remains the leading cause of death worldwide. Anti-platelet agents are found improve the patient outcomes even-though it carries risk of bleeding which needs to be carefully monitored. 
The relative contribution (efficacy) of drugs used in anti-platelet therapy always remained a question. From this study we conclude that ticagrelor revealed better efficacy than clopodogrel in terms of NSTEMI and Ejection Fraction.

\section{Compliance with ethical standards}

\section{Acknowledgments}

The authors thank staff of DurgabaiDeshmukh hospital for their kind support they gave to undertake and complete this study successfully.

\section{Disclosure of conflict of interest}

The authors declare no conflict of interest.

\section{Statement of ethical approval}

Study was conducted with the approval of Institutional ethics committee, DurgabaiDeshmukh hospital, Hyderabad.

\section{Statement of informed consent}

Informed consent was obtained from all individual participants included in the study.

\section{References}

[1] Saleh M, Ambrose JA.(2019). Understanding myocardial infarction. F1000 Research, 7:1000 Faculty Rev, 1378.

[2] Thomas FLüscher. (2015). Myocardial infarction mechanisms, diagnosis, and complications. European Heart Journal, 36(16), 947-949.

[3] Ezra A. Amsterdam, Nanette K. Wenger, Ralph G. Brindis, Donald E. CaseyJr, Theodore G. Ganiats et.al. (2014). 2014 AHA/ACC Guideline for the management of patients with non-ST-Elevation acute coronary syndromes. Circulation, 130(25), 344-426.

[4] Maxwell S. (1999). Emergency management of acute myocardial infarction. British journal of clinical pharmacology, 48(3), 284-298.

[5] Kernan WN, Ovbiagele B, Black HR, Bravata DM, Chimowitz MI, Ezekowitz MD, et al. (2014). Guidelines for the prevention of stroke in patients with stroke and transient ischemic attack. Stroke, 45(7), 2160-2236.

[6] Greaves SC. (2002). Role of echocardiography in acute coronary syndromes. Heart, 88(4), 419-425.

[7] Massberg S, Polzin A. (2018). Dual Antiplatelet Therapy. Dtsch. Med. Wochenschr, 143(15), 1090-1093.

[8] Komosa A, Lesiak M, Siniawski A, Mularek-Kubzdela T, Grajek S. (2014). Significance of antiplatelet therapy in emergency myocardial infarction treatment. Advances in interventional cardiology, 10(1), 32.

[9] Alizadehasl A, Sadeghpour A, Behjati M. (2017). The role of echocardiography in acute myocardial infarction. Indian heart journal, 69(4), 563.

[10] Layne K, Ferro A. (2017). Antiplatelet Therapy in Acute Coronary Syndrome. European cardiology, 12(1), 33-37. 